\title{
Influência da Distância Intereletrodos e da Cadência de Movimento no Domínio da Frequência do Sinal de EMG de Superfície
}

\section{Influence of the Inter-Electrodes Distance and Cadence of Movement in the Surface EMG Signal Frequency Domain}

Flávia Fernandes Tank

Gustavo Telles da Silva

Carlos Gomes de Oliveira

Marco Antonio Cavalcanti Garcia

Laboratório de Biomecânica -

EEFD/UFRJ.

\section{Endereço para correspondência:}

Marco Antonio Cavalcanti Garcia

Laboratório de Biomecânica -

EEFD/UFRJ

Rua Maranhão, 305, casa 5 - Meier

20720-230 - Rio de Janeiro, RJ

E-mail: marcoacg_ufrj@hotmail.com

Submetido em 19/10/2008

Versão final recebida em 26/04/2009

Aceito em 05/05/2009

\begin{abstract}
RESUMO
A eletromiografia de superfície (sEMG), apesar de amplamente utilizada em investigações biomecânicas, ainda apresenta inúmeros questionamentos sobre a influência das distâncias intereletrodos (DIE) na morfologia do sinal, principalmente em contrações isotônicas. Logo, muitos dos trabalhos desenvolvidos ainda se limitam ao âmbito do laboratório de pesquisa, onde é possível estabelecer maior controle nos protocolos de registro e análise, o que não é comumente observado na prática clínico-desportiva. Dessa forma, o objetivo do estudo foi examinar os efeitos de dois protocolos de colocação de eletrodos e a realização de contrações isotônicas no domínio da frequência do sinal de sEMG. Quinze sujeitos do sexo masculino (idade: $22,8 \pm 3,5$ anos), todos destros, realizaram contrações dinâmicas do bíceps braquial direito com carga estimada em $20 \%$ da contração voluntária máxima em três diferentes cadências $(30,45,60 \mathrm{bpm})$. Os sinais de sEMG foram registrados por meio de dois canais, cujas DIEs foram de 4,2 e 13cm, respectivamente. A avaliação dos sinais de sEMG foi baseada na frequência mediana do espectro de potencial do sinal, calculado via transformada rápida de Fourier. A DIE e a cadência foram definidas como fatores (ANOVA two-way; $\alpha=0,05$ ). Não foram observadas diferenças estatísticas e qualquer interação entre ambos os fatores nas três cadências $(P>0,05)$. Sugere-se que, independentemente da distância utilizada entre os eletrodos, uma investigação no domínio da frequência do sinal de sEMG em tarefas dinâmicas seja evitada, mesmo a partir de DIEs reduzidas, como é sugerido pela literatura, dado que variações no torque e no comprimento muscular podem corromper o sinal e, portanto, sua interpretação.
\end{abstract}

Palavras-chave: eletromiografia, processamento digital de sinais, contração isotônica.

\section{ABSTRACT}

Surface electromyography (SEMG), despite being widely used in biomechanical investigations, still presents massive questioning about the influence of the distance of the inter-electrodes (DIE) in the signal morphology, especially in isotonic contractions. Thus, much of the research developed is still limited to the laboratory, where it is possible to establish better control over the recording and analysis protocols, which is not commonly observed in the clinical-sportive practice. Therefore, the aim of this study was to examine the effects of two electrodes placement protocols and the performance of isotonic contractions in the SEMG sign frequency domain. Fifteen right-handed male subjects (aged $22.8 \pm 3.5$ years) performed dynamic contractions of the right brachial biceps with load estimated in $20 \%$ of the maximum voluntary contraction in three different cadences $(30,45$ and $60 \mathrm{bpm})$. The SEMG signals were registered by two channels with DIEs of 4.2 and $13 \mathrm{~cm}$, respectively. The SEMG signals assessment was based on the median frequency of the potential spectrum of the signal, calculated via fast Fourier transform. DIE and cadence were defined as factors (two-way ANOVA; $\alpha=0.05$ ). No statistical differences or any interaction between both factors were observed in the three cadences ( $P>0.05$ ). Regardless of the distance used between electrodes, an investigation in the SEMG signal frequency domain in dynamic tasks should be avoided, even from reduced DIEs, as suggested in the literature, since variations in the torque and muscular length may disrupt the signal and hence its interpretation.

Keywords: electromyography, digital signal processing, isotonic contraction. 


\section{INTRODUÇÃO}

Hamill e Knutzen discutem que o tecido muscular esquelético, motor primário de nossa interação com o ambiente, age de forma complexa mesmo em tarefas que acreditamos serem extremamente simples ${ }^{(1)}$. Logo, tentar compreender as estratégias de ativação muscular em qualquer que seja essa tarefa demanda não somente conhecimentos de anatomia, mas também da biomecânica e do controle motor envolvido. Uma forma amplamente utilizada na investigação da ação muscular é a eletromiografia (EMG). A EMG é definida como uma técnica que trata da detecção e análise do sinal elétrico que provém da contração do tecido muscular esquelético ${ }^{(2)}$. De acordo com Hermens et al. ${ }^{(3)}$, houve aumento significativo do conhecimento em torno dessa técnica, principalmente na década de 1990; tendo em vista o crescente número de trabalhos publicados, a Sociedade Internacional de Eletrofisiologia e Cinesiologia (ISEK)(3) passou a estabelecer normas de aquisição e tratamento do sinal de EMG, inclusive quanto à colocação de eletrodos de superfície, um dos tipos de transdutores comumente utilizados.

Pullman et al.(4) descrevem que, em algumas ocasiões, os eletrodos de EMG de superfície (sEMG) são inapropriados para a aquisição do sinal de EMG. Face e mão são exemplos de regiões de difícil acesso no monitoramento da atividade mioelétrica por meio de eletrodos de superfície. Nesses casos, o uso de eletrodos invasivos (agulha ou arame) muitas vezes se faz necessário. Eletrodos invasivos também são sugeridos quando há a possibilidade de somação de sinais de músculos vizinhos com a atividade do músculo alvo, o que é definido como crosstalk ${ }^{(3)}$. Kossev e Christova ${ }^{(5)}$ lembram que a estabilidade física do eletrodo de arame também contribui para melhor registro do sinal de EMG durante a realização de movimentos repetidos. Christensen et al. ${ }^{(6)}$ também relatam que o uso da sEMG leva a risco de mudanças no posicionamento de eletrodos durante a realização de movimentos, o que pode causar mudanças na composição espectral do sinal de sEMG e induzir a interpretação equivocada dos mecanismos de recrutamento muscular. Nesses casos, a ISEK sugere que a distância intereletrodos não ultrapasse $20 \mathrm{~mm}$ centro a centro, de forma que não se perca grande parte da informação contida no sinal pelo movimento dos sensores sobre o músculo ${ }^{(3)}$.

Ainda assim, desde que levadas em consideração algumas das orientações sugeridas pela ISEK, o sinal de EMG colhido na superfície da pele pode ser usado para estudar funções musculares durante tarefas que envolvam contrações isométricas e isotônicas; também tem sido usada para examinar aspectos de ordem central e periférica da fadiga neuromuscular, na estratégia de ativação de unidades motoras e na modulação da produção do torque (ou força) $)^{(7)}$.

Além dos aspectos relacionados com o processo de aquisição, outro importantíssimo assunto diz respeito aos procedimentos utilizados na extração de parâmetros do sinal de sEMG, que pode ser analisado nos domínios do tempo e/ou frequência ${ }^{(7)}$. No domínio do tempo, analisa-se a energia do sinal por meio de parâmetros correlacionados com sua amplitude, a qual reflete os mecanismos de gradação da força muscular (recrutamento de unidades motoras - UMs - e a somação de seus potenciais de ação). Já no domínio da frequência, busca-se determinar a contribuição de funções periódicas (senos e cossenos) na composição do sinal de sEMG, algo que não é trivial em termos de relação com os processos de gradação da força muscular. Dentre os métodos existentes, o mais utilizado é a transformada de Fourier, um método que permite decompor o sinal de sEMG em componentes espectrais ${ }^{(3)}$. Nesse domínio são estabelecidas relações com a velocidade de condução dos potenciais de ação, assim como a taxa de disparos das mesmas pelas unidades motoras ${ }^{(2,8)}$. No que se refere às informações colhidas a partir da decomposição espectral do sinal, Beck et al. ${ }^{(8)}$ relatam que, no caso da frequência mediana, esta é fortemente correlacionada com a velocidade média de condução dos potenciais de ação na fibra muscular, o que permite avaliar alguns fenômenos como fadiga muscular e doenças neuromusculares ${ }^{(9)}$.

No entanto, Elfving et al.(10) destacam que os parâmetros extraídos no domínio da frequência são sensíveis à forma pela qual os eletrodos são posicionados sobre o músculo, havendo, portanto, correlação negativa entre as frequências média e mediana do sinal de sEMG com a distância intereletrodos. Desse modo, o aumento na distância produz variação na composição do espectro de potência do sinal para baixas frequências. Sugere-se, então, que as orientações publicadas pela ISEK(3) sejam seguidas sempre que possível, de forma que os grupos de pesquisa em sEMG troquem informações, permitindo comparações ${ }^{(8)}$. No caso da distância intereletrodos, Hermens et al. ${ }^{(3)}$ apontam que uma distância limite de $20 \mathrm{~mm}$ entre os eletrodos também poderá ajudar a reduzir o efeito de crosstalk, dado que distâncias maiores aumentam a probabilidade de o sinal de sEMG ser contaminado pela atividade de músculos vizinhos ${ }^{(8,11)}$. Por outro lado, alguns estudos têm indicado que o aumento da distância entre eletrodos aumenta a profundidade com que os eletrodos detectam o potencial de ação, ampliando a contribuição dos potenciais de ação das unidades motoras no sinal de $E M G^{(11,12)}$. Logo, conclui-se que uma variação na distância entre os eletrodos interferirá na morfologia do sinal, não somente no domínio da frequência, mas também da amplitude, comprometendo a interpretação dos mecanismos de gradação da força muscular ${ }^{(7)}$.

Outra importante orientação diz respeito ao local de colocação dos eletrodos de superfície. Hermens et al. ${ }^{(3)}$ e Malek et al..$^{(7)}$ discutem que os eletrodos devem ser posicionados sobre o ventre muscular e entre a zona de inervação e a região tendinosa, caso contrário, o sinal de sEMG poderá também ter sua morfologia alterada. Dessa forma, estudos prévios têm sugerido que a amplitude do sEMG é maximizada quando os eletrodos são colocados sobre o ventre muscular, entre a zona de inervação e a região tendinosa e com distância limite de $20 \mathrm{~mm}$. Outros estudos também citam que o posicionamento dos eletrodos sobre ou perto da zona de inervação resulta em baixa amplitude na sEMG e altos valores de frequência do sinal quando comparado com a colocação de eletrodos entre a zona de inervação e regiões tendinosas do músculo(13,14,15).

Dessa forma, o objetivo deste estudo foi verificar o comportamento da variabilidade do sinal de sEMG a partir da frequência mediana do espectro de potência do sinal, em três diferentes cadências de contração, e a partir de dois protocolos distintos de colocação de eletrodos de superfície, comumente utilizados nas práticas clínica e desportiva, no músculo bíceps braquial.

\section{MÉTODOS}

Participaram do estudo 15 sujeitos do sexo masculino (idade: $22,8 \pm$ 3,6 anos; massa corporal: 75,5 1 11,6kg; estatura: 1,76 \pm 0,09m), destros, e sem histórico de lesões em membros superiores e coluna lombar. Todos os sujeitos eram universitários e praticantes de atividade física. Todos assinaram o Termo de Consentimento Livre e Esclarecido submetido e aprovado pelo Comitê de Ética em Pesquisa da instituição ( $n$ o 125/03). Para avaliar a dominância de membros superiores foi aplicado o Edinburgh Handedness Inventory ${ }^{(16)}$.

Para aquisição dos sinais de sEMG foi utilizado um eletromiógrafo ME3000P de oito canais (Mega Eletronics, Ltd., Finlândia) e um computador portátil (Pentium II) (figura 1). Os sinais foram coletados à taxa de $1 \mathrm{kHz}$ e ganho de 1.000. Foram utilizados eletrodos de superfície de Ag/AgCl (3M Korea Ltd., 27-3, Seul, Coreia; 1cm de diâmetro) e um metrônomo digital GMT-200P (Groovin USA Design) para controle da cadência de movimento (figura 2). 


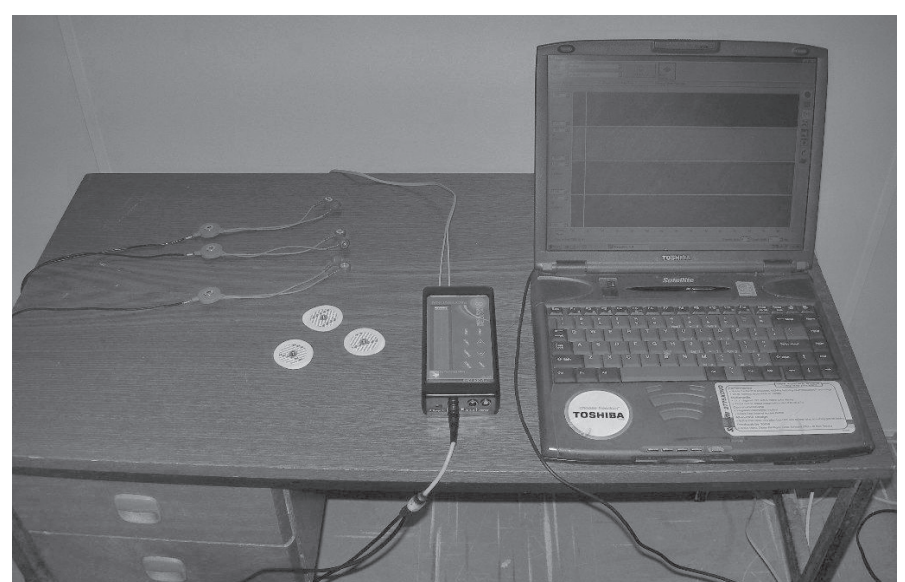

Figura 1. Sistema de aquisição de sinais de sEMG

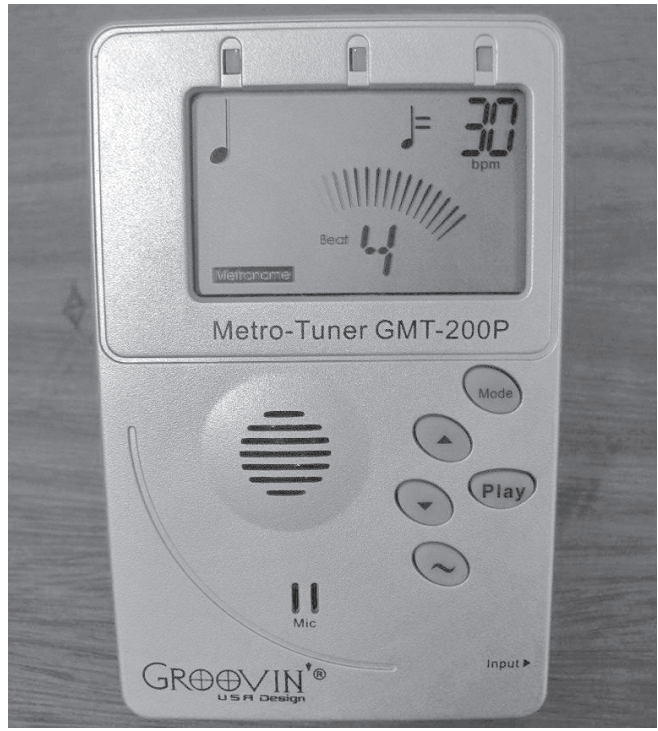

Figura 2. Metrônomo digital

A atividade mioelétrica foi registrada a partir do ventre do músculo bíceps braquial direito, por meio de dois pares de eletrodos em configuração bipolar. Um par, mais interno (C1), apresentou distância de 4,2cm e o outro, mais externo (C2), distância de $13 \mathrm{~cm}$ (figura 3). Essas distâncias foram assim definidas, pois os eletrodos não foram propositalmente recortados. Logo, a dimensão dos mesmos foi respeitada, conforme pode ser observado na figura 3. A região de colocação dos eletrodos foi previamente preparada, mediante tricotomia e lavagem com água e sabão. O protocolo de colocação dos eletrodos foi realizado de acordo com a proposta SENIAM ${ }^{(3)}$. Para isso foi preciso identificar os seguintes pontos anatômicos: acrômio e fossa cubital. Sabida a distância entre esses pontos, foi estimada uma medida de 30\% a partir do segundo ponto na direção do primeiro. Essa serviu como ponto médio para a colocação dos pares de eletrodos. Os eletrodos foram colocados sobre a linha determinada entre os pontos anatômicos supracitados, respeitando a direção das fibras musculares. Os eletrodos de referência foram colocados o mais próximo possível dos epicôndilos medial e lateral do úmero.

Os sujeitos foram informados sobre os procedimentos do teste e treinados para a realização das contrações isotônicas do bíceps braquial. Para a realização do teste, foi utilizado protocolo de aquisição com base em três diferentes cadências de realização de movimento, cujas velocidades médias se aproximaram de 750, 112,50 e 150\%/segundo, controladas por meio de um metrônomo que serviu como referência para os sujeitos (30,45,60bpm). A ordem das cadências foi determinada por meio de sorteio.

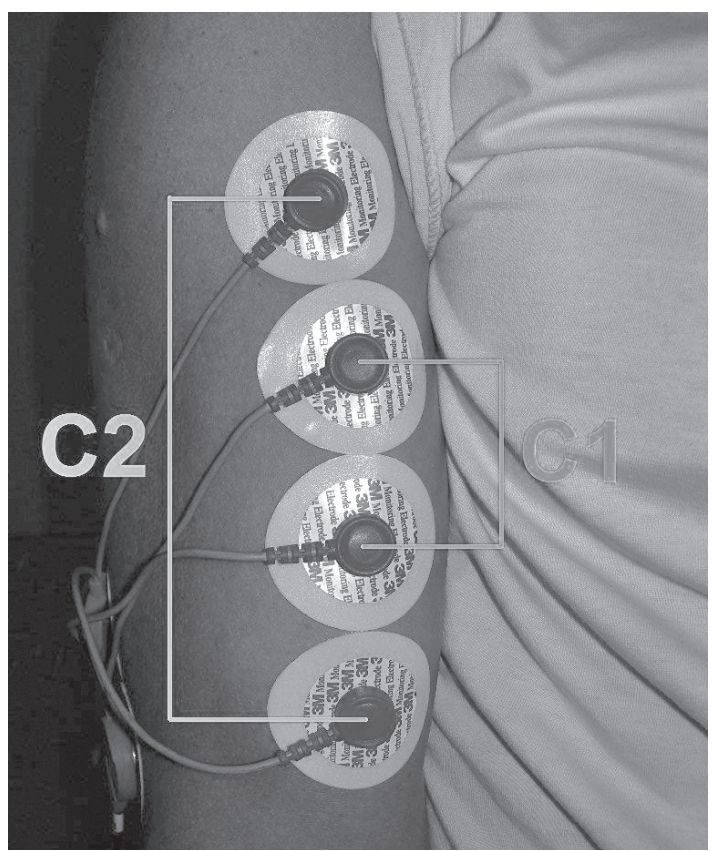

Figura 3. Protocolo de colocação de eletrodos. C1- Par interno e C2 - Par externo.

Para a realização dos testes, o indivíduo encontrava-se em posição ortostática, pernas alinhadas com os ombros, cabeça em posição neutra, joelhos semiflexionados e mão esquerda fixada à cintura (figura 4). A execução do teste se deu por meio de três contrações concêntricas e excêntricas dos flexores do cotovelo, com carga estimada em 20\% da carga máxima (CM), conforme descrito a seguir.

Os avaliadores mediram massa, estatura, dobra cutânea do tríceps e perímetro do braço, ambos do membro superior direito. O perímetro do braço foi medido no ponto médio entre o acrômio e o olécrano, estando os indivíduos em posição ortostática e com o membro superior direito relaxado e estendido. A medição da dobra cutânea do tríceps foi realizada seguindo a padronização proposta por Pollock, segundo Pompeu et al.(17). Para mensuração da massa corporal e da estatura foi utilizada uma balança mecânica (Filizola, resolução de 100g). Para o perímetro do braço utilizou-se uma trena metálica e, para dobra cutânea, um plicômetro (Slim Guide, resolução de 1mm).

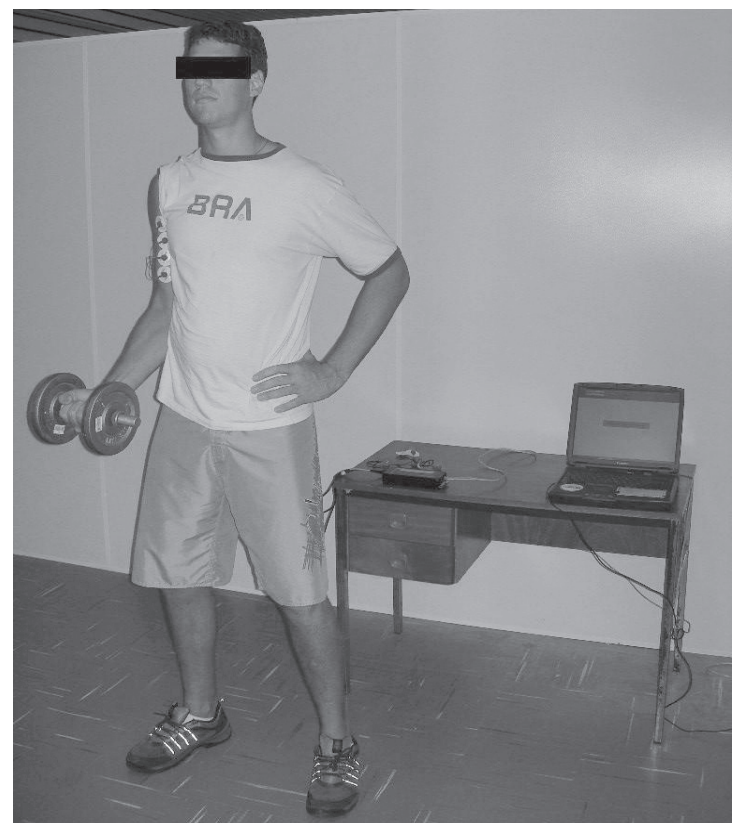

Figura 4. Posição adotada pelo sujeito para realização dos testes de contração 
Obtidas essas medidas, foi utilizada a equação 1 para estimativa da área de secção transversa muscular do braço $\left(\mathrm{AM}_{\mathrm{B}}\right)^{(17)}$.

$A_{M B}=\frac{[C-(T \times \pi)]^{2}}{(4 \times \pi)}$

Onde:

$\mathrm{A}_{\mathrm{MB}}$ representa a área de secção transversa total do braço $\left(\mathrm{cm}^{2}\right)$;

C é perímetro do braço $(\mathrm{cm})$;

Té a dobra cutânea tricipital $(\mathrm{cm})$; e

$\pi$, constante igual a $3,14 \ldots$;

Determinada a área de secção transversa total ocupada pelos músculos flexores e extensores do cotovelo, o passo seguinte foi extrair aquela representada somente pela parte flexora. Dessa forma, a seguinte equação (2) foi aplicada:

$A_{f}=A_{M B} \times 0,356$

Onde:

$A_{f}$ representa a área dos músculos flexores do braço;

$A_{m b}$ representa a área de secção transversa total do braço $\left(\mathrm{cm}^{2}\right)$;

e 0,356, área relativa dos flexores primários (bíceps e o braquial).

Determinada a área dos músculos flexores, o passo seguinte foi calcular a força dos músculos flexores primários do braço. A seguinte equação (3) foi estabelecida:

$F_{f}=63 \times A_{f}$

Onde:

$F_{f}$ é a força dos flexores primários do braço;

63 é a tensão específica dos flexores (Newton/cm²)(18); e

$A_{f}$ representa a área dos músculos flexores do braço.

Em seguida, o último passo foi estimar a capacidade máxima de geração de força dos músculos flexores primários do braço. A seguinte equação (4) foi estabelecida:

$$
C_{m}=\frac{\left(F_{F}\right) \times 3,5}{B_{R}}
$$

Onde:

$\mathrm{C}_{\mathrm{m}}$ significa carga máxima;

$F_{f}$ é a força dos flexores primários do braço;

3,5 é o comprimento da alavanca dos flexores do cotovelo (cm); e $B_{r}$ é o braço de resistência (epicôndilo lateral ao processo estiloide do rádio).

Após esses procedimentos foi possível estimar a CVM de cada sujeito.

A marcação dos trechos de sEMG para análise foi realizada a partir da utilização de um cursor (disponível no software). Os trechos de interesse selecionados foram os intermediários e de forma arbitrária (ex:: havendo cinco contrações concêntricas e excêntricas, a terceira era a escolhida). A partir desses trechos, eram marcados o início e o fim do burst (figura 5). Portanto, não foi possível adquirir janelas (T) de mesmo tamanho, sendo esta uma das limitações do estudo. Para essas janelas foi aplicada a transformada de Fourier e a frequência mediana calculada conforme a seguinte equação (5):

$$
\int_{0}^{f m} S m(f) d f=\int_{f m}^{\infty} S m(f) d f
$$

Onde:

$S_{m}$ é o espectro de potência do sinal de EMG; e fm a frequência mediana.

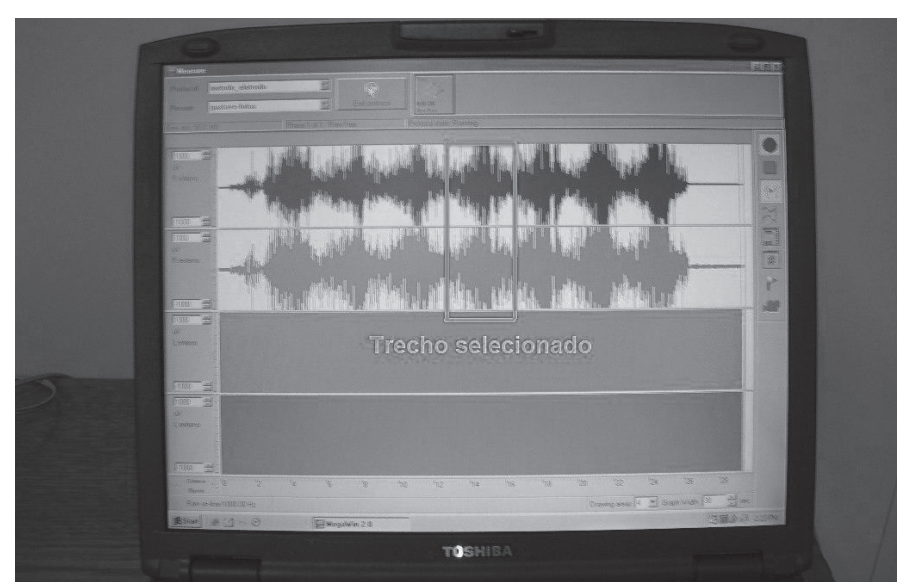

Figura 5. Exemplo do processo de seleção do burst de interesse

Como os sinais de sEMG foram colhidos em condições dinâmicas, o registro poderia apresentar maior variabilidade quando comparado com contrações do tipo isométrico. Logo, a escolha da mediana deveuse às propriedades estatísticas da medida, que não é sensível a valores extremos, diferentemente da média.

Para a análise estatística foi utilizada a ANOVA two-way, cujos fatores foram "cadência" e "distância intereletrodos" (C1 e C2). Portanto, foram comparadas as médias entre as três cadências e as distâncias C1 e C2. O nível de significância (a) adotado foi de 0,05.

\section{RESULTADOS}

A Figura 6 apresenta os resultados dos valores médios da três diferentes cadências e os valores médios da frequência mediana do sinal de sEMG, entre C1 e C2, para as contrações dinâmicas do músculo bíceps braquial.

Apesar de C2 $(13 \mathrm{~cm})$ apresentar valores de frequência mediana do sinal de sEMG superiores aos expressos por C1 (4,2cm), não foi observada diferença estatística significativa $\left(F_{(1,84)}=0,37939 ; P=0,5396\right)$ entre ambos os protocolos nas três cadências.

Na comparação entre as três cadências, foi observada tendência à diminuição do valor médio da frequência mediana do sinal de sEMG, conforme o aumento das mesmas nas contrações isotônicas. Contudo, não foi observada diferença estatística significativa $\left(F_{(2,84)}=0,37203\right.$; $P=0,69046)$ entre as três cadências. Além disso, não foi observada interação entre a distância e cadência na modulação da frequência mediana do sinal de sEMG.

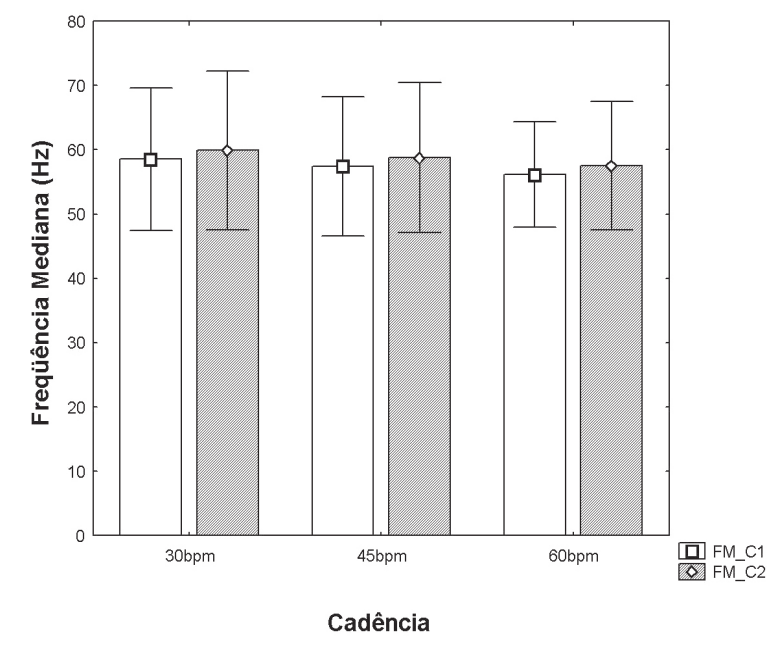

Figura 6. Valores médios ( \pm DP) da fm de C1 e C2 nas três cadências (30, 45 e 60bpm). 


\section{DISCUSSÃO}

Supunha-se inicialmente que haveria um efeito significativo da distância intereletrodos sobre a fm. No entanto, neste presente estudo não foi observada diferença estatística significativa entre as distâncias dos dois canais (C1 e C2), o que corrobora parcialmente os achados de Malek et al. ${ }^{(7)}$ e Beck et al. (8). Estes observaram que, quando os dados da sEMG são normalizados, não ocorre diferença significativa na distância intereletrodos. Ao contrário do proposto por esses autores, no presente estudo os dados da fm do sinal de sEMG foram analisados de forma bruta e não normalizados.

Outro importante aspecto foi que, como os eletrodos utilizados neste estudo são facilmente adquiridos no mercado e não foram propositalmente cortados para se ajustar à distância mínima exigida pelas normas SENIAM ${ }^{(3)}$, acredita-se que a distância utilizada entre os eletrodos tenha sido o principal fator no padrão de comportamento da fm, ou seja, mesmo para um músculo de fácil acesso como o bíceps braquial, a menor distância adotada $(4,2 \mathrm{~cm})$ talvez tenha sido suficientemente grande para produzir efeitos de distorção do sinal e comprometimento do parâmetro escolhido. Logo, não somente foi inviável qualquer interpretação dos mecanismos de gradação da força muscular envolvidos na tarefa escolhida, como também na busca pelos efeitos do fator "distância intereletrodos" na fm.

Um estudo realizado por Beck et al. ${ }^{(8)}$ comparou os efeitos das distâncias intereletrodos $(2,4$ e $6 \mathrm{~cm})$ na frequência mediana do sinal de sEMG durante ações musculares isocinéticas e isométricas do bíceps braquial e observou que as mudanças nas três diferentes distâncias intereletrodos também não conduziu a diferenças na fm em relação aos torques dinâmico e isométrico, corroborando parcialmente os resultados aqui apresentados. Por outro lado, outro estudo realizado por Malek et al. ${ }^{(7)}$ também discutiu os efeitos da distância intereletrodos (2, 4 e $6 \mathrm{~cm}$ ) na fm do sinal de sEMG do vasto lateral durante a pedalada em um cicloergômetro e observou que,com o aumento da mesma, havia redução desse parâmetro. Quando os dados da fm do sinal de sEMG foram normalizados, as diferenças médias foram eliminadas.

Em uma ampla revisão na literatura sobre os aspectos relativos à colocação de eletrodos de superfície sobre a musculatura paravertebral, Elfving et al. ${ }^{(10)}$ discutem que a mudança da distância intereletrodos de 2 para $4 \mathrm{~cm}$ pode causar diminuição significativa na frequência média do espectro do sinal de sEMG, de aproximadamente $20 \%$, no músculo eretor da espinha ao nível de L3. Esses mesmos autores também afirmam que pode haver diminuição significativa desse mesmo parâmetro, em aproximadamente 10\%, quando a distância intereletrodos muda de 3,5 para 5,5cm, no músculo eretor da espinha ao nível de L4. Todavia, Elfving et al. ${ }^{(10)}$ discutem que em outro estudo metodológico não foram observadas diferenças significativas na frequência média quando a distância intereletrodos mudou de 2,5 para 3,5cm para o músculo eretor da espinha aos níveis de T10 e L3. Tais observações corroboram a idéia de que

\section{REFERÊNCIAS BIBLIOGRÁFICAS}

1. Hamill J, Knutzen KM. Bases Biomecânicas do Movimento Humano. 1a Ed. São Paulo: Editora Manole, 1999.

2. De Luca CJ. Electromyography. Encyclopedia of medical devices e instrumentation. 2a Ed. Hoboken: John Wiley \& Sons, Inc., 2006.

3. Hermens JH, Freriks B, Klug CD, Rau G. Development of recommendations for SEMG sensors and sensor placement procedures. J Electromyogr Kinesiol 2000;14:361-74.

4. Pullman SL, Goodin DS, Marquinez Al, Tabbal S, Rubin M. Clinical utility of surface EMG. Neurol 2000;55:171-7.

5. Kossev A, Christova P. Discharge pattern of human motor units during dynamic concentric and eccentric contractions. Electroencephalogr Clin Neurophysiol 1998;109:245-55.

6. Christensen H, Sogaard K, Jensen BR, Finsen L, Sjogaar G. Intramuscular and surface EMG power spectrum from dynamic and static contractions. J Electromyogr Kinesiol 1995;5:27-36.

7. Malek MH, Housh TJ, Coburn JW, Weir JP, Schmidt RJ, Beck TW. The effects of interelectrode distance on electromyographic amplitude and mean power frequency during incremental cycle ergometry. J Neurosci Methods 2006:151:139-47.

8. Beck TW, Housh TJ, Johnson GO, Weir JP, Cramer JT, Coburn JW, Malek MH. The effects of interelectrode distance on electromyographic amplitude and mean power frequency during isokinetic and isometric muscle actions of the biceps brachii. J Electromyogr Kinesiol 2005;15: 482-95.

9. Kamen G, Caldwell GE. Physiology and interpretation of the electromyogram. J Clin Neurophysiol 1996;13:366-84

10. Elfving B, Liljequist D, Mattsson E, Németh G. Influence of interelectrode distance and force level on the spectral parameters of surface electromyographic recordings from the lumbar muscles. J Electromyogr Kinesiol 2002;12:295-304. a arquitetura muscular - tendo em vista os diferentes níveis da coluna torácica e lombar - também pode ser uma importante variável na morfologia do sinal de sEMG e, portanto, de seu conteúdo em frequência.

Kamen e Caldwell(9) discutem que pequenas distâncias intereletrodos estão comumente associadas com grandes larguras de bandas de frequência e, portanto, maiores valores médios. Com base nesses diferentes estudos é possível observar que o conteúdo espectral do sinal de sEMG não somente pode variar com a distância, bem como o tipo de músculo investigado. Isso porque a disposição das fibras musculares e o comprimento das mesmas em relação aos eletrodos também poderá produzir modulações no sinal de sEMG(3).

Farina e Mesin ${ }^{(13)}$ e Roy et al.(15) discutem também que eletrodos localizados perto ou sobre a zona de inervação, condição provável deste estudo, conduzem a maiores valores médios de frequência. Entretanto os achados de Beck et al.(19) não corroboram os achados de Farina e Mesin ${ }^{(13)}$ e Roy et al. ${ }^{(15)}$, onde foi observado que os eletrodos posicionados perto ou sobre a zona de inervação não resultam em grandes valores absolutos da frequência média do sinal de sEMG.

\section{CONCLUSÃO}

Este estudo teve como objetivo analisar a influência da distância intereletrodos $(4,2$ e $13 \mathrm{~cm})$ na fm do sinal de sEMG do músculo bíceps braquial em três cadências distintas de movimento da articulação do cotovelo.

Como foi visto, foram utilizados eletrodos sem recortes, o que explica as grandes distâncias intereletrodos adotadas. Logo, supõe-se que ambos os pares de eletrodos tenham "transitado" sobre as zonas de inervação e tendinosas durante a realização da tarefa, comprometendo, assim, a qualidade do sinal analisado. Portanto, é possível que o parâmetro escolhido para esta análise não apresente qualquer relação com os mecanismos envolvidos na regulação da contração muscular da tarefa proposta. No entanto, acreditando-se que a menor distância adotada (4,2cm) estivesse sobre o ventre muscular durante toda a amplitude de movimento, os resultados ratificam as normas SENIAM ${ }^{(3)}$, de que em condições dinâmicas o sinal de sEMG apresentaria maiores níveis de não estacionariedade, o que desqualificaria sua análise a partir do domínio da frequência. Tal condição reduziria drasticamente qualquer possibilidade de inferência sobre, por exemplo, recrutamento e/ou modulação em frequência de unidades motoras a partir de parâmetros tais como a fm nesses casos.

Recomenda-se, então, que, independentemente da distância utilizada entre os eletrodos, uma investigação no domínio da frequência do sinal de sEMG em tarefas dinâmicas seja evitada na medida em que, variações no torque e no comprimento muscular, podem corromper o sinal e, portanto, sua análise.

Todos os autores declararam não haver qualquer potencial conflito de interesses referente a este artigo.
11. Farina D, Mesin L. Sensitivity of surface EMG-based conduction velocity estimates to local tissue inhomogeneities - influence of the number of channels and inter-channel distance J Neurosci Methods 2005;142:83-89.

12. Mercer JA, Bezodis N, Delion D, Zachry T, Rubley MD. EMG sensor location: Does it influence the ability to detect differences in muscle contraction conditions? J Electromyogr Kinesiol 2006;16:198-204.

13. Farina D, Colombo R, Merletti R, Olsen HB. Evaluation of intra-muscular EMG signal decomposition algorithms J Electromyogr Kinesiol 2001;11:175-87.

14. Rainoldi A, Nazzaro M, Merletti R, Farina D, Caruso I, Gaudenti S. Geometrical factors in surface EMG of the vastus medialis and lateralis muscles J Electromyogr Kinesiol 2000;10:327-36.

15. Roy SH, De Luca CJ, Schneider J. Effects of electrode location on myoelectric conduction velocity and median frequency estimates. J Appl Physiol 1986;61:1510-17.

16. Oldfield RC. The assessment and analysis of handedness: the Edinburgh inventory. Neuropsychologia 1971;9:97-113.

17. Pompeu FAMS, Gabriel D, Pena BG, Ribeiro P. Áreas de secção transverso do braço: implicações técnicas e aplicações para avaliação da composiçăo corporal e da força dinâmica máxima. Rev Bras Med Esporte 2004;10:202-06.

18. Kawakami Y, Nakazawa K, FujimotoT, Nozaki D, Miyashita M, Fukunaga T. specific tension of elbow flexor and extensor muscles based on magnetic resonance imaging. Eur J appl Physiol Occup Physiol 1994;68:139-47.

19. Beck TW, Housh TJ, Cramer JT, Weir JP. The effects of electrode placement and innervation zone location on the electromyographic amplitude and mean power frequency versus isometric torque relationships for the vastus lateralis muscle. J Electromyogr Kinesiol 2008;18:317-28. 